\title{
Physical Activity Training for Functional Mobility in Older Persons
}

\author{
Tom Hickey \\ Fredric M. Wolf \\ Lynne S. Robins \\ University of Michigan \\ Marilyn B. Wagner \\ Cleveland State University \\ Wafa Harik \\ Case Western Reserve University
}

The effectiveness of low-intensity physical activity for improving functional ability and psychological well-being in chronically impaired older individuals was demonstrated in a pilot study. Participants who completed 6 weeks of structured low-intensity exercise $(\mathrm{N}=77)$ improved in the time and number of steps required to walk a measured course, in self-assessments of mobility and flexibility, and in three measures of well-being. Those who continued to exercise in a peer-led program $(\mathrm{n}=32)$ maintained improvements in mobility and optimism after 18 weeks.

Difficulties in balance and mobility and limitations in musculoskeletal strength, range of motion, and flexibility are fairly common among older people. For example, $19 \%$ of noninstitutionalized individuals age 65 years and over experience difficulty in walking, and approximately $8 \%$ have difficulty in getting in and out of chairs and beds (National Center for Health Statistics, 1987). Among individuals between the ages of 75 and 84 who were followed in the Framingham Disability Study, 39\% were unable to stand for a 15 -minute period, $23 \%$ were unable to walk one-half mile, $15 \%$ could not climb stairs, $24 \%$ were unable to lift small items (up to 10 pounds), and more than $50 \%$ were unable to lift heavier items or to stoop, crouch, or kneel without difficulty or assistance (Jette \& Branch, 1981). Because these find-

\footnotetext{
AUTHORS' NOTE: This research was supported by a grant from the Cleveland Foundation and conducted in collaboration with the Golden Age Centers of Greater Cleveland, Inc., Cleveland, $\mathrm{OH}$.
} 
ings were based on a relatively healthy older sample, the magnitude of such disability problems in the older population may be even greater.

These deficits in strength, range of motion, and flexibility contribute to the loss of functional independence, placing frail older people at risk for institutionalization. In fact, limited mobility is a critical risk factor associated with health dependency among older people. Difficulties in walking and related physical problems are significant determinants of the need for institutional care (Branch \& Jette, 1982; Weissert \& Cready, 1989).

There is evidence to suggest that losses in functional mobility can be prevented in many older persons through physical activity. Various forms of exercise have been found to improve strength and mobility and to increase cardiac fitness necessary for active functioning (Holloszy, 1983; Lampman \& Savage, 1988; Morey et al., 1991; Vallbona \& Baker, 1984). Given the importance of functional status for independence in the older population, the specific role of different types of physical activity in maintaining or improving the physical and functional dimensions of mobility requires close examination in older adults at high risk of institutionalization.

This report summarizes a preliminary investigation of the effectiveness of low-intensity exercise for improving the functional health of older adults with limited mobility as a result of chronic health problems and prolonged physical inactivity. The data reported in this article were collected in a research demonstration intended (a) to develop a low-intensity exercise intervention that would appeal to chronically impaired older persons and be relatively easy for them to do and (b) to provide a basis for planning further research to determine whether light exercise could lead to measurable functional health benefits.

The exercise intervention was called SMILE, for So Much Improvement with a Little Exercise. The SMILE program was suggested by an earlier study of low-intensity exercise on people with diabetes, which demonstrated that structured physical activity could improve functional health and mobility in chronically impaired and sedentary adults (Howard et al., 1985). The SMILE program employs a similar nonaerobic, low-intensity exercise intervention. However, it was refocused to emphasize movements that address the specific limitations in strength, range of motion, and mobility of physically inactive older persons for whom high-intensity exercise was not a realistic option. The intervention also was intended to minimize the risks associated with undertaking a physical activity regimen for sedentary older persons with chronic impairments in functional mobility.

Although numerous studies have investigated various health-related effects of exercise, most of what is known about the outcomes of physical activity on the health and functioning of older persons is based on relatively 
healthy populations, and most interventions are of short duration and of moderate to high intensity, such as aerobic programs, where effects are evident after a short period of time. Only a few studies have investigated the functional health benefits of low-intensity activity (Gossard et al., 1986; Harkom, Lampman, Banwell, \& Castor, 1985). With some exceptions (e.g., Morey et al., 1991; Thompson, Crist, Marsh, \& Rosenthal, 1988), little is known about what beneficial outcomes might be obtained from a sustained program of low-intensity physical activity for older people. The effects of low-intensity exercise carried out over a longer period of time may be at least as effective as high-intensity exercise of a shorter duration. In one study, for example, differences between the effects of high- and low-intensity exercise on aerobic capacity and blood lipids did not begin to disappear until 18 weeks of training (Gaesser \& Rich, 1984). In other words, the low-intensity program merely took longer to have essentially the same effects.

The impact of exercise on older persons has been measured largely in terms of changes in heart rate or blood pressure or of overall health benefits such as weight reduction. Fewer studies have examined the specific effects of structured physical activity on musculoskeletal movement, joint flexibility, and balance and gait, which are essential for functional mobility and performance of the basic activities of daily living (ADL). Participation in physical activities designed to address these factors needs to be evaluated in more functionally impaired older persons than have been studied previously to establish the potential efficacy of exercise interventions for older people at high risk of chronic disability.

Determining the functional health outcomes of low-intensity exercise on the performance of basic ADL in a frail older population, however, represents a considerable challenge. In addition to the health and safety risks of involving frail individuals in a physical activity intervention, initiating and maintaining an exercise program is especially difficult for physically inactive persons who are limited by chronic impairments and who are typically less motivated to participate in any type of structured exercise program. The present study was designed to address these challenges.

\section{Method}

\section{Sample}

Participants for this study were drawn from the client registry of a nonprofit human services agency serving older people in senior centers affiliated with public housing facilities in a large urban area. Through the use 
of newsletter announcements, informational posters, and individual contacts by staff and peers, individuals from three senior centers were recruited to participate in the exercise program. Individuals were excluded on the basis of recent major surgery, myocardial infarction, or stroke. Persons with serious medical problems requiring major daily management or monitoring were also excluded. The 90 participants who began the program were mostly female $(94 \%)$, White $(52 \%)$ or Black (48\%), and living alone $(61 \%)$, with an average age of 72.6 years. The dropout rate was consistent with similar exercise programs. At the end of 18 weeks, the sample size $(N=32)$ was slightly below what is required for adequate power estimates $(N=34)$. This will be addressed in a follow-up study.

Most participants described their own health as only fair (44\%) or poor (36\%), and more than $66 \%$ expressed concern about their health and functioning. Participants reported an average of three chronic conditions that caused some impairment. All participants reported arthritis, hypertension, and related heart disease. Most of these physically inactive older people were overweight (average Body Mass Index > 28.6) and had not previously participated in an exercise program.

\section{Measures}

All assessments were conducted in random order at the senior center site of the exercise intervention within 2 weeks of the beginning and end of the program, taking no more than 30 minutes for each participant. Functional mobility was assessed in two ways. First, participants rated their abilities to perform basic ADL in five areas. Using the Functional Status Index (FSI), mobility (five items), personal care (four items), home chores (three items), hand activities (three items), and social roles (five items) were self-assessed (yes/no) according to whether they caused pain or difficulty or required assistance in carrying them out. Total scores for the five functional areas were derived from the number of yes responses for pain, difficulty, and assistance on each item in that domain. Thus the possible scores ranged from 0 to 15 for mobility, from 0 to 12 for personal care, from 0 to 9 for home chores, from 0 to 9 for hand activities, and from 0 to 15 for social roles. The FSI is a self-assessment of functioning that has demonstrated substantial construct validity and high reliability in studies of older persons with osteoarthritis and related functional mobility problems (Jette, 1980). Levels of internal consistency range from .66 to .91 , and test-retest and interobserver reliabilities are .70 or better.

A performance assessment of walk time and number of steps taken over a measured course was also conducted as an additional measure of flexibility 
and balance in mobility. Participants were asked to walk a 20-meter course, which entailed walking 10 meters in one direction, turning around, and walking back to the starting point. Each individual was timed, and the number of steps taken was counted by one of two different raters. Interrater reliability was .94 .

Assessment of psychological well-being was suggested by other studies of older samples that have found both physical and mental health outcomes from different types of exercise (Emery \& Blumenthal, 1990; Kaplan, Atkins, \& Remsch, 1984; King, Taylor, \& Haskell, 1989). Based on some of our earlier work, we selected three measures for this study to assess the impact of a low-intensity exercise intervention on different dimensions of psychological well-being as a basis for further research in this area. Participants were asked to respond to 12 dichotomous items (agree/disagree) on the Life Orientation Test (LOT), which measured future outlook and optimism as a basis for predicting behavioral outcomes (Scheier \& Carver, 1985). Potential scores could range between 0 and 12. A five-item Attitude Toward Aging factor (agree/disagree) contained in the Morale Scale (Lawton, 1975) was used to assess the impact of aging on participants' daily lives. Thus the range of possible scores was 0 to 5 .

Finally, based on other studies that have suggested the importance of measuring expectations about the efficacy of participating in an exercise program (e.g., Kaplan et al., 1984), an exercise-based self-efficacy scale was developed specifically for this study to assess participants' beliefs about their own abilities to perform five of the SMILE exercises successfully (i.e., foot circles, forward bends, arm flexes, shoulder rolls, and marching in place). This measure consisted of five items, each scored on a 3-point Likert-type scale, where $1=$ not at all sure, $2=$ somewhat sure, and $3=$ very sure. Total scores could range between 5 and 15 . For all three measures, higher scores indicated a more positive outlook. Evidence of the validity of our selfefficacy measure was supported by a pattern of predictable validity coefficients with other measures in this study. For example, higher levels of baseline self-efficacy were associated with lower levels of ADL functioning $(r=-.22$, $p<.05$, two-tailed test) but with higher levels of morale $(r=.18)$ and optimism $(r=.10)$. These latter correlations were in the predicted direction but were low and suggest that self-efficacy as measured in the present study is a distinct construct and measures something different from morale or optimism.

Participants were asked two open-ended questions about the impact of the program on overall health and on their experience of muscle and joint pain. They were also asked whether they planned to continue to participate in the SMILE program with peer leaders. 


\section{Procedure}

The SMILE program consisted of repeated stretching, flexibility, and range of motion exercises based on their potential to strengthen muscle and joint activity, to improve postural balance, and to enhance the performance of basic activities essential for maintaining independent functioning (e.g., standing, sitting, walking, reaching, stooping, and hand/finger flexibility). Specifically, the SMILE program combined 25 movements into a 30-minute set routine that began and ended with diaphragmatic breathing for relaxation. The initial sequence was done in a seated position and involved 7 gentle movements for the neck, shoulders, and hands, followed by 10 upper body exercises done in a standing position. These included various reaching and stretching exercises designed to promote shoulder flexibility and arm strength as well as spine twists, side stretches, and pelvic rotations for trunk flexibility and walking balance. This sequence was followed by 7 lower body exercises done in a seated position to strengthen stomach and pelvic muscles and to increase flexibility and range of motion in the hips (e.g., leg lifts, pelvic tilts, hip hikes/rotations, and leg swings). Two ankle exercises were also performed at this point to develop strength and coordination and to improve circulation in the feet. The next segment of the SMILE routine involved 5 exercises in a standing position to develop flexibility and strength in the lower body for such activities as climbing stairs or rising out of a chair (e.g., arm curls, heel lifts, leg swings, and side/back leg stretches). The first of two cool-down routines was done seated and at a slower pace. This routine involved gentle stretching movements for the upper body including shoulder stretches, forward bends to increase spine flexibility, posture exercises, and arm and finger movements for range of motion in arm, wrist, and finger joints. The final routine involved gentle stretching and flexing movements of the legs and knees, arms, and shoulders as well as full body stretching in a standing position. The number of repetitions of each movement was based on the level of difficulty of the movement (see Hickey, Wolf, Robins, Wagner, \& Harik, 1992, and Hickey, Luchowski, \& Wolf, 1994, for a complete description of the SMILE program).

The SMILE intervention was conducted twice weekly in four groups of 20 to 25 participants. After the first 6 weeks, the two professional instructors began to encourage the participants to take turns leading short segments in each exercise session as an initial step toward empowering the group members to exercise on their own. Individualized training was then provided for 8 peer leaders who were selected by the participants to conduct the SMILE program following the departure of the professional instructors. During the 
final 6 weeks, peer leaders gradually assumed leadership for the SMILE program, with the professionals providing technical assistance and advice. Participants were assessed prior to beginning the SMILE program, following the 6-week demonstration, and at the end of 18 weeks.

\section{Results}

Of the initial 90 participants, $77(86 \%)$ were still in the program and available for assessment following 6 weeks. The number of participants declined to $32(35 \%)$ at the end of 18 weeks. Average weekly attendance was just under $70 \%$ for all groups and remained fairly stable. For purposes of analysis, individuals were treated as participants on the basis of attending at least two thirds of the sessions. Although this rate is somewhat arbitrary, it was suggested by earlier work of Morey et al. (1989). The participant group was easily identified: Those who missed two or three consecutive sessions tended to drop out of the program entirely; those who remained were fairly consistent in their participation, with only an occasional absence. Healthrelated problems were the primary reasons given for dropping out or missing sessions, and there were no differences in the number of chronic illnesses between those who continued to exercise and those who dropped out. No one was injured or experienced any adverse health effects as a direct result of their participation in the SMILE program. After 6 weeks, more than $85 \%$ of the participants reported feeling better than when they had started, and $80 \%$ reported a decrease in the daily experience of muscle and joint pain. Almost all of the participants (97\%) indicated their intent to continue exercising regularly.

\section{Exercise Effects}

As indicated in Table 1, following 6 weeks of low-intensity exercise, there was an improvement in participants' assessments of mobility $(p<.00)$, which included walking, climbing stairs, rising from a chair, reaching overhead, and stooping or crouching. Participants also reported increased flexibility in hand movements $(p<.03)$ related to writing, opening a jar or bottle, and dialing a telephone. Mean scores were based on the number of items with which participants reported difficulty, pain, and/or the need for assistance. Thus the maximum score was 15 for both mobility and social role activities (each of which had five items), 12 for personal care (four items), and 9 for both home chores and hand activities (three items each). Higher mean scores reflected 
Table 1. Self-Assessments of Functional Performance and Observations of Selected Gait Parameters Between Basellne and 6-Week Follow-Up $(N=77)$

\begin{tabular}{|c|c|c|c|c|c|c|}
\hline & \multicolumn{2}{|c|}{ Baseline } & \multicolumn{2}{|c|}{ 6-Week Follow-Up } & \multirow[b]{2}{*}{$\mathrm{t}$} & \multirow[b]{2}{*}{$p$} \\
\hline & Mean & $\overline{S D}$ & Mean & SD & & \\
\hline \multicolumn{7}{|l|}{ Functional measure } \\
\hline Mobility & 5.04 & 3.11 & 3.60 & 3.22 & 4.87 & .00 \\
\hline Personal care & 0.34 & 0.08 & 0.29 & 0.81 & 0.52 & .60 \\
\hline Home chores & 1.83 & 2.54 & 1.40 & 2.47 & 1.54 & .13 \\
\hline Hand activities & 0.95 & 1.77 & 0.57 & 1.06 & 2.21 & .03 \\
\hline Social role activities & 1.38 & 3.14 & 1.04 & 2.33 & 0.91 & .37 \\
\hline \multicolumn{7}{|l|}{ Gait parameter } \\
\hline Walk time & 8.66 & 15.63 & 7.32 & 15.81 & 2.92 & .01 \\
\hline Number of steps & 11.40 & 4.69 & 10.10 & 2.80 & 2.41 & .02 \\
\hline
\end{tabular}

Table 2. Analysis of Variance Results of Psychological Assessments Between Baseline and 6-Week Follow-Up $(N=77)$

\begin{tabular}{|c|c|c|c|c|c|c|}
\hline & \multicolumn{2}{|c|}{ Baseline } & \multicolumn{2}{|c|}{ 6-Week Follow-Up } & \multirow[b]{2}{*}{$F$} & \multirow[b]{2}{*}{ p } \\
\hline & Mean & SD & Mean & SD & & \\
\hline \multicolumn{7}{|l|}{ Measure } \\
\hline Optimism & 5.62 & 1.61 & 6.25 & 1.43 & 14.85 & .00 \\
\hline Morale & 3.57 & 1.62 & 4.06 & 1.16 & 6.75 & .00 \\
\hline Self-efficacy & 14.03 & 1.50 & 14.45 & 1.08 & 4.85 & .01 \\
\hline
\end{tabular}

more pain/difficulty/need for assistance, and lower scores indicated greater independence in these functions. Performance assessments of mobility showed significant improvements in actual walk time $(p<.01)$ and decreases in the number of steps in normal gait used to cover a measured course $(p<.02)$.

Table 2 summarizes the outcomes of psychological assessments of optimism, morale, and self-efficacy. As indicated in higher mean scores, participants improved significantly across all three dimensions after 6 weeks of the SMILE intervention.

\section{Maintenance Effects}

Self-assessments and performance observations were conducted after 18 weeks to determine whether initial improvements would be sustained in a peer-led maintenance program. As indicated in Table 3, those who continued in the SMILE program $(N=32)$ maintained their initial improvements in 


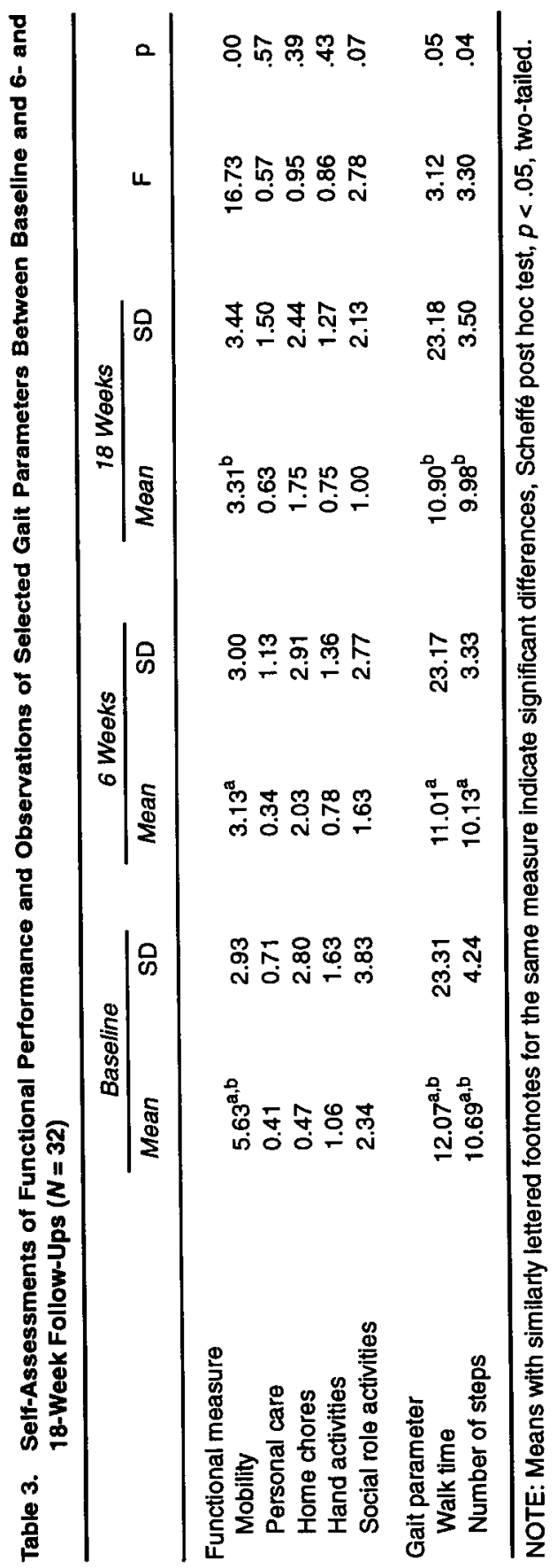


self-assessments and in performance measures of mobility. Their selfassessments of pain, difficulty, and/or need for assistance in carrying out various social role activities (i.e., getting into and out of a vehicle, going to and from the senior center, and moving about the community including shopping and visiting friends) were also more positive. The improvements in well-being found at the end of the first 6 weeks were maintained in their 18-week scores only on the optimism scale (see Table 4). Given the attrition rate, these findings may not be representative of the larger group assessed at baseline.

\section{Discussion}

The results of this pilot study are indicative of the potential efficacy of low-intensity physical activity for improving mobility in frail and functionally impaired older persons in at least two ways. First, this project demonstrated that exercise interventions can be designed to address the limitations and interests of physically inactive older people with considerable chronic impairment. Second, as a result of participation in an intervention that was designed to enhance the physical movement skills needed by older people to maintain their independent functioning, there was improvement in selfreported assessments of functional ability in carrying out activities of daily living and in mobility as gauged by two simple evaluations. These preliminary findings regarding mobility are encouraging for the development of controlled interventions to evaluate more specific functional health effects of this type of intervention on the performance of basic ADL by frail older persons.

The continuing participation in a peer-led program at the conclusion of this research by more than one third of the initial group suggests that previously sedentary individuals with considerable frailty can be encouraged to increase and sustain their levels of physical activity. This finding, which is based on a group of older people more impaired than those studied by either Thompson et al. (1988) or Morey et al. (1989), suggests the importance of targeting exercise interventions and maintenance programs specifically for elderly patients with significant physical and functional impairments.

These findings are necessarily preliminary and descriptive, in that participants remaining at the end of 18 weeks may have been a more select group. Also, a greater number of participants are necessary to provide sufficient statistical power to demonstrate improvement over time. In addition, health maintenance effects are more difficult to evaluate in the absence of controlled 


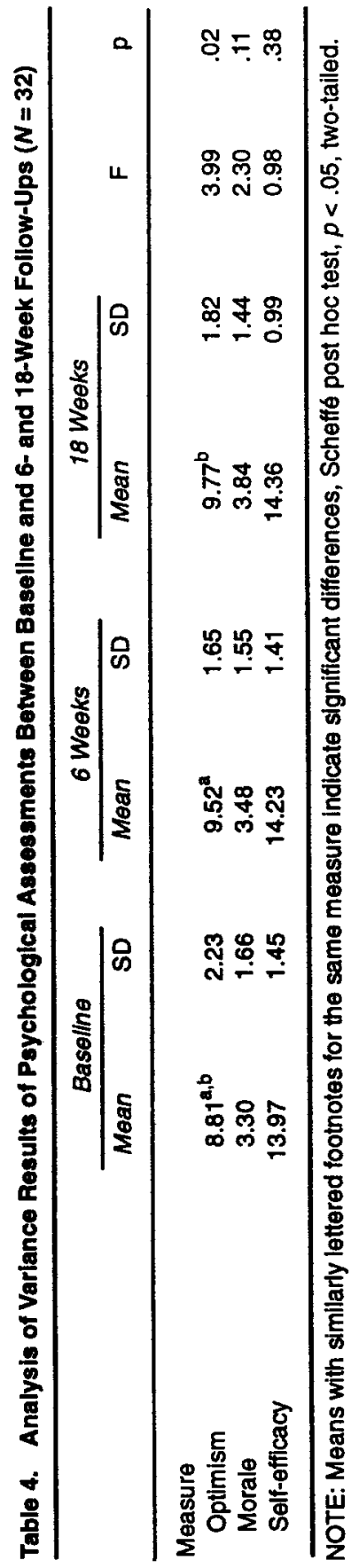


observations of the impact of the different peer leaders on participation and program adherence.

The study was further limited by the single-group design in that the initial group of participants may have been more motivated to exercise. The two brief performance measures of walking behavior may have also been confounded by practice effects and motivational factors. Despite these limitations, the results of this pilot investigation are an encouraging indication of the types of functional benefits that frail older individuals might derive from participating in limited physical activity programs.

Careful clinical trials using additional performance measures along the line suggested by Tinetti and Ginter (1988) are now required to validate these results in older persons with different levels and types of impairments. Further research also will be important to determine the relationship of specific physical activity interventions to a broad range of functional mobility outcomes and to the performance of basic ADL. There is no reason to expect, for example, that improvements will be uniform across individuals with differing impairments or in all dimensions of mobility: For example, greater gains may be possible with some older individuals in hand, arm, or upper body flexibility than in ambulation, balance, or gait.

However limited the results, performance assessments of basic mobility functions appear to be sensitive measures of the effects of structured physical activity on functional mobility. For example, two simple observational measures seemed to capture small improvements in walking behavior, and related observations of other components of balance and gait were also positive, although not significant. Additional clinical assessments of rangeof-motion flexibility were also conducted with a subgroup of exercise participants to test the feasibility and validity of such measures for future research. These participants generally sustained or improved their range-ofmotion flexibility in neck, trunk, shoulder, and ankle movements, which are essential for successful balance and gait. Careful assessments at more frequent intervals are necessary to indicate the impact of the physical activity intervention on other dimensions of mobility than the limited gait parameters measured here. An important next step will be to observe and quantify the dimensions of change in the performance of specific physical movements that contribute to overall functional mobility.

Although participation in the SMILE program appeared to have a positive impact on overall well-being, this should be considered a very preliminary finding. Further research is needed to delineate more precisely the interaction of psychological factors with both participation and health outcomes. At the same time, the positive effects shown here should not be ignored. Reports of 
reduction in muscle and joint pain, which were an early and consistent result of the intervention, may have been an important factor in the mental health benefits observed. The sense of mastery participants derived from doing the exercises may have contributed to the overall positive psychological outcomes as well. Participants were consistently enthusiastic about the SMILE sessions, as demonstrated by these typical comments: "Coming here gives me so much energy" and "This program makes me feel good."

\section{Conclusion}

This pilot study has provided useful information for the development of practice interventions and of further applied research by demonstrating the potential health effects of low-intensity physical activity in a sample of relatively frail older persons. It is consistent with related studies that have shown that the initial physical and functional benefits derived from an exercise intervention with frail older persons can be maintained (Morey et al., 1991). However, it goes beyond other investigations by identifying what types of physical activity may lead to improvements in mobility and flexibility in physically inactive and chronically impaired older people. This study suggests that exercise interventions that both appeal to physically inactive older people who are limited in functional mobility and demonstrate positive health outcomes in a chronically impaired older population can be designed. Although these findings are based on a preliminary descriptive study, they suggest that low-intensity physical activity may have longer term effects on various dimensions of functional mobility and overall health status.

\section{References}

Branch, L. G., \& Jette, A. M. (1982). A prospective study of long-term care institutionalization among the aged. American Journal of Public Health, 24(4), 485-510.

Emery, C. F., \& Blumenthal, J. A. (1990). Perceived change among participants in an exercise program for older adults. The Gerontologist, 30(2), 184-188.

Gaesser, G. A., \& Rich, R. G. (1984). Effects of high and low-intensity exercise training on aerobic capacity and blood lipids. Medicine and Science for Sports and Exercise, 16, 269-274.

Gossard, D., Haskell, W. L., Taylor, C. B., Mueller, J. K., Rogers, F., Chandler, M., Ahn, D. K., Miller, N. H., \& DeBusk, R. F. (1986). Effects of low and high-intensity home-based exercise training on functional capacity in healthy middle-aged men. American Journal of Cardiology, $57,446-449$. 
Harkom, T. M., Lampman, R. M., Banwell, B. F., \& Castor, C. W. (1985). Therapeutic value of graded aerobic exercise training in rheumatoid arthritis. Arthritis and Rheumatology, 28(1), 32-38.

Hickey, T., Luchowski, A. T., \& Wolf, F. M. (1994). Low-risk exercise for high-risk older women. The Female Patient, 19, 47-54.

Hickey, T., Wolf, F. M., Robins, L. S., Wagner, M. B., \& Harik, W. (1992). So much improvement with a little exercise [videotape with manuals]. Ann Arbor: University of Michigan School of Public Health.

Holloszy, J. O. (1983). Exercise, health and aging; A need for more information. Medicine and Science for Sports and Exercise, 15, 1.

Howard, M., Funnell, M., Robins, L., Johnson, T., Lampman, R. M., \& Wolf, F. (1985). Development and evaluation of a low-level exercise program for diabetics. Diabetes, 34(Suppl. 1), 133A.

Jette, A. M. (1980). Functional Status Index: Reliability of a chronic disease evaluation instrument. Archives of Physical and Medical Rehabilitation, 61, 395-401.

Jette, A. M., \& Branch, L. G. (1981). The Framingham disability study: Physical disability among the aging. American Journal of Public Health, 71(11), 1211-1216.

Kaplan, R. M., Atkins, C. J., \& Remsch, S. (1984). Specific efficacy expectations mediate exercise compliance in patients with COPD. Health Psychology, 3, 223-242.

King, A. C., Taylor, C. B., \& Haskell, W. L. (1989). Influence of regular exercise on psychological health: A randomized, controlled trial of healthy middle-aged adults. Health Psychology, 8 , 305-324.

Lampman, R. M., \& Savage, P. J. (1988). Exercise and aging: A review of benefits and a plan for action. In J. R. Sowers \& J. V. Felicetta (Eds.), The endocrinology of aging (pp. 307-335). New York: Raven.

Lawton, M. P. (1975). The Philadelphia Geriatric Center Morale Scale: A revision. Journal of Gerontology, 30, 85-89.

Morey, M. C., Cowper, P. A., Feussner, J. R., DiPasquale, R. C., Crowley, G. M., Kitzman, D. W., \& Sullivan, R. J., Jr. (1989). Evaluation of a supervised exercise program in a geriatric population. Journal of the American Geriatrics Society, 37, 348-354.

Morey, M. C., Cowper, P. A., Feussner, J. R., DiPasquale, R. C., Crowley, G. M., \& Sullivan, R. J., Jr. (1991). Two-year trends in physical performance following supervised exercise among community-dwelling older veterans. Journal of the American Geriatrics Society, 39 , 549-554.

National Center for Health Statistics. (1987). Aging in the 80's: Functional limitations of individuals age 65 and over. Washington, DC: U.S. Department of Health and Human Services.

Scheier, M. F., \& Carver, C. S. (1985). Optimism, coping, and health: Assessment and implications of generalized outcome expectancies. Health Physiology, 4(3), 219-247.

Thompson, R. F., Crist, D. M., Marsh, M., \& Rosenthal, M. (1988). Effects of physical exercise for elderly patients with physical impairments. Journal of the American Geriatrics Society, $36,130-135$.

Tinetti, M., \& Ginter, S. F. (1988). Identifying mobility dysfunctions in elderly patients. Journal of the American Medical Association, 259, 1190-1193.

Vallbona, C., \& Baker, S. B. (1984). Physical fitness prospects in the elderly. Archives of Physical and Medical Rehabilitation, 65, 194-200.

Weissert, W. G., \& Cready, C. M. (1989). Toward a model for improved targeting of aged at risk of institutionalization. Health Services Research, 24(4), 485-510. 
Tom Hickey is a professor in the Department of Health Behavior and Health Education at the University of Michigan School of Public Health and a faculty associate at the Institute of Gerontology in Ann Arbor, MI. His current research is directed at improving mobility in chronically impaired older adults. He is a coauthor of "The Attribution of Health Problems to Aging and Mortality Among Older Adults in a Longitudinal Study" (American Journal of Public Health, 1992) and of "Adaptation of a General Optimism Scale for Use With Older Women" (Psychological Reports, 1994).

Fredric $M$. Wolf is a professor of postgraduate medicine and health professions education and the director of the Learning Resource Center and Laboratory for Computing, Cognition and Clinical Skills at the University of Michigan Medical School. His research interests include the evaluation, dissemination, and adoption of new technology, decision making and judgment, and the social-psychological aspects of chronic illness. He is the author of Meta-analysis: Quantitative Methods for Research Synthesis (Sage, 1986).

Lynne S. Robins is an assistant professor in the Department of Postgraduate Medicine and the director of curriculum evaluation at the University of Michigan Medical School. Her research interests include physician-patient interactions and the influence of gender and ethnicity on health care delivery and on medical education. She is a coauthor of "The Effect of Training on Medical Students' Responses to Geriatric Patient Concerns: A Linguistic Analysis" (The Gerontologist, 1989).

Marilyn B. Wagner is a physical therapist in the Department of Health Sciences at Cleveland State University. Her teaching and research are focused on movement science and exercise with adult populations at special risk.

Wafa Harik is a nurse who was affiliated with the School of Nursing at Case Western Reserve University at the time this research was conducted. She directs exercise interventions for functionally impaired older adults. 